\title{
DEGREES OF SYMMETRY IN QUANTUM FIELD THEORIES
}

\author{
Th.A.J.MARIS, D.DILLENBURG*, G.JACOB* and B.LIBERMAN \\ Instituto de Fisica and Faculdade de Filosofia** \\ Universidade Federal do Rio Grande do Sul \\ Porto A legre, Brasil
}

Received 9 November 1970

(Expanded version received 7 July 1971)

\begin{abstract}
A hierarchy of possible symmetries in quantum field theory is defined, which reaches from a purely mathematical invariance to the conventional physical invariance, including the commonly discussed type of spontaneously broken symmetry (SBS). It is shown that one type of SBS, which is usually not considered, naturally leads to theories with an algebra of non-conserved currents and a non-linearly transforming phenomenological Lagrangian. An exactly solvable model is given and some general remarks are made.
\end{abstract}

\section{INTRODUCTION}

After it became evident that there exist certain approximate symmetries which are very useful for the understanding of leptons and hadrons, the question naturally arose whether at some time one might be able to derive qualitatively or even quantitatively the observed deviations from the exact invariances. It is clear that to do this, one will have to start from a theory in which no specific deviation from the symmetry in question is a priori preferred, i.e., from a symmetric theory. The asymmetry would then necessarily have to be generated by the dynamics itself. This is the bootstrap idea of the spontaneously broken symmetry (SBS) theories.

In the applications $[1-3]$ of this idea to quantum field theory, there soon emerged a principle difficulty. From Noether's theorem it follows that a symmetric theory should contain a conserved symmetry current and it can be shown [4-7] under rather general conditions, that the symmetry can only be spontaneously broken if the zero component of this conserved current applied on the vacuum state creates a massless boson. As a rule however, these Goldstone bosons (or the equivalent long range interactions) are not observed and, in fact, also the meas-

* Senior Research Fellow, Conselho Nacional de Pesquisas.

** Work supported by Banco Nacional do Desenvolvimento Econômico, U.S. Army Research Office, Conselho Nacional de Pesquisas, Regional Program of Scientific and Technological Development of the OAS and Conselho de Pesquisas UFRGS. 
urable symmetry currents are not conserved. To reproduce these observations it is common use $[2,8]$ to also break the symmetry of the original theory externally. This procedure however abandons the main motivation of the whole undertaking,:namely the hope to be able to derive the asymmetries from the theory.

Fortunately there exist some arguments which suggest that there must be a weak point in the chain of reasoning which leads from a symmetric field theory to conserved currents and Goldstone bosons. Consider, for example, dilatational invariance. It is not difficult to see, and we shall repeat the argument in sect. 3 , that the arbitrariness in the choice of the unit of length implies that a complete theory of physics, if it exists at all, could be formulated as one in which the dilatational invariance is spontaneously broken $[9,10]$. Nevertheless in nature the dilatational current is not conserved and also no corresponding Goldstone boson occurs. Now it is, of course, quite possible that nature is not describable by a field theory, but the quantum electrodynamics (QED) of massless electrons and muons [10-12] shows that in fact dilatational invariance can be spontaneously broken in a field theory in just the observed way. In this theory there occurs also a $U_{2} \otimes U_{2}$ symmetry group containing several other spontaneously broken invariances $[13,14]$ with non-conserved symmetry currents. Here one might have the objection that this model has been only very approximately treated and that in an exact calculation a conserved symmetry current could emerge. For this reason we will give later an exactly solvable (albeit somewhat trivial) model of a relativistic local quantum field theory in which a spontaneously broken symmetry is generated by a non-conserved current.

In this paper we shall attempt to show (for earlier remarks in the same spirit see refs. $[5,15,16])$ that the difficulty of conserved currents and Goldstone bosons in SBS theories is a "self-inflicted agony" [17]. The bootstrap idea of spontaneous symmetry breaking in a quantum field theory does not demand the conservation of the symmetry current; on the contrary, the by definition singular properties of the field operators render it nearly impossible for the current conservation to survive the breaking of the vacuum symmetry $[18,19]$ and make the classical Noether's theorem inapplicable. A very special condition, which is the statement of Goldstone's theorem, is necessary (and not even sufficient) for the current to remain conserved if the vacuum symmetry is lost.

In sect. 2 we define the concept of passive symmetry. With the help of this definition we are able to generalize the usual notion of a spontaneously broken symmetry. Then by imposing increasingly stronger conditions, one obtains a hierarchy of symmetries which extends from a purely mathematical invariance to the usual "good" one and which includes the commonly discussed type of SBS. Examples of each of these cases are known. In sect. 3 we give an exactly solvable model and a discussion of the type of SBS which we believe to be relevant for elementary particle physics. Finally some more general remarks are made.

In the following a certain familiarity with the conventional theory of spontaneous symmetry breaking is assumed $[3,5,20,21]$; it is not our intention to at- 
tempt a selfcontained and rigorous or even systematic treatment, but we only wish to bring out certain physical points which have not received much attention in the literature and which we believe to be essential.

\section{A HIERARCHY OF SYMMETRIES}

In this section we consider some classes of SBS solutions which can occur. It should however be kept in mind that in any well defined physical theory the type of solution which is in fact realized is not subject to ones choice, but is determined by the dynamics.

We first define the concept of a passive symmetry: We shall say that a quantum theory of fields $\psi_{i}$ has a passive invariance (symmetry) if there exists an algebraic transformation on the set of all field operators

$$
\psi_{i} \rightarrow \psi_{i}^{\prime}\left(\psi_{j}\right)
$$

which transforms any solution $\psi_{i}$ of the theory (given as an irreducible representation of the field algebra in a Hilbert space) into a new solution $\psi_{i}^{\prime}$ of the theory.

It may be noted that we have not specified any detail of the theory (Lagrangian, field equation, etc.) and that our criterion eliminates possibilities of confusion inherent in certain other definitions where, for example, the question may arise whether a theory is only "formally invariant" but not actually so because necessary limiting procedures destroy the "invariance" [18]. The decisive question for the presence of a passive invariance is only whether one can show that there exists a transformation (1) which generates a new solution.

We shall give in sect. 3 a simple example in which the existence of a certain passive symmetry is easy to see. In the more realistic cases in which there are infinite or finite renormalizations involved, it is in general necessary to take in to account that the renormalization itself depends on the solution of the theory and therefore transforms along with the field under transformation (1). Of course, nowhere in the theory (including the renormalization procedure) may occur any condition which selects a priori one special solution in the set of solutions defined by transformation (1); this would by definition destroy the passive invariance. We will also give an example of this realistic type of passive invariance in sect. 3 . It is clear that the presence of a certain passive invariance puts a condition on the renormalization procedure and this is in itself of interest.

A passive invariance is a property of the mathematical formulation of a theory. However, if a passive invariance transformation is represented by a unitary operator in the Hilbert space of the solution, it is just a usual good physical symmetry, as for example Lorentz invariance in general is.

Definition: We call a passive symmetry spontaneously broken in a solution of the theory, when the transformation (1) can not be represented by a unitary operator in the Hilbert space of the solution. 
In the following we shall only consider passive invariance transformations which depend on a continuous parameter and which are local in the basic fields, essentially meaning that the transformed field operators $\psi^{\prime}$ for each space-time point $x^{\prime}$ only depend on fields $\psi$ in one point $x\left(x^{\prime}\right)$. The function $x\left(x^{\prime}\right)$ is assumed to be linear and to represent a Lorentz transformation and/or a dilatation; the special conformal transformation is briefly discussed in sect. 4 .

If only the above mentioned conditions are fulfilled, the so defined SBS has no great physical contents. It is realized in certain solutions of the Schwinger model as discussed in ref. [22].

Consider now an arbitrary space-like plane and let us suppose that it is possible to supplement the basic field operators $\psi(x)$ by a set of field operators $\pi(x)$, constructed from the field operators $\psi$, such that each set $(\psi(x), \pi(x))$ with $x$ in the plane, is complete in the sense of Schur's lemma. In a canonical theory the operators $\pi$ could, for example, be the canonical momenta. Because of their construction, the transformation (1) of the basic field operators induces now also a transformation on the fields $\pi$ and one has the relation

$$
\{\psi ; \pi\} \rightarrow\left\{\psi^{\prime}(\psi) ; \pi^{\prime}(\psi, \pi)\right\},
$$

which is assumed to be still local.

Suppose further that we have a special type of SBS, namely one in which a local current $j^{\mu}(x)$ exists, which generates the transformation $\left(1^{\prime}\right)$, in the sense of ref. [6], if applied on a complete set $(\psi(y), \pi(y))$ with all $y$ in a spacelike plane through the point $x$. In an equal time plane, for example, one has

$$
\lim _{V \rightarrow \infty}\left[\int_{V} j^{\mathrm{o}}\left(\boldsymbol{x}, x_{\mathrm{o}}\right) \mathrm{d}^{3} x, \phi\left(y, x_{\mathrm{o}}\right)\right]=-\left.i \frac{\partial \phi^{\prime}\left(\boldsymbol{y}, x_{\mathrm{o}}\right)}{\partial \alpha}\right|_{\alpha=0},
$$

$\alpha$ denoting the parameter of the continuous passive transformation and $\phi$ being any algebraic expression containing only $\psi$ 's and $\pi$ 's in the plane $t=x_{0}$ and $\phi$ ' being the same expression in $\psi^{\prime}$ and $\pi^{\prime}$. For an arbitrary plane the current component orthogonal to the plane should be taken instead of $j^{\mathrm{o}}$.

It is important to note that relation (2) is only demanded for each fixed $x_{0}$ (in general for each space-like plane) common to the charge and to the complete set of basic field operators. If, for example, an operator $\partial \psi / \partial x_{0}$ does not belong to this set then in general

$$
\lim _{V \rightarrow \infty}\left[\int_{V} j^{\mathrm{o}}\left(\boldsymbol{x}, x_{\mathrm{o}}\right) \mathrm{d}^{3} x, \frac{\partial \psi\left(\boldsymbol{y}, x_{\mathrm{o}}\right)}{\partial x_{\mathrm{o}}}\right] \neq-\left.i \frac{\partial}{\partial \alpha} \frac{\partial \psi^{\prime}\left(\boldsymbol{y}, x_{\mathrm{o}}\right)}{\partial x_{\mathrm{o}}}\right|_{\alpha=0},
$$

because for $\psi$ in another plane a different charge is needed to generate transformation $\left(1^{\prime}\right)$. Therefore, in this case the passive transformation ( $\left(1^{\prime}\right)$ is an algebraic isomorphism for the quasi-local subalgebras spanned by the basic sets of field operators $(\psi, \pi)$ in corresponding space-like planes, but it will in general not be an 
automorphism for the whole algebra of quasi-local fields. For essentially this reason we shall call this case the one in which the symmetry is locally broken [15].

What follows for a physical system described by a solution of the present type?

a) The commutation rules (2) for a complete set of operators essentially define the charge

$$
\lim _{V \rightarrow \infty} \int j \mathrm{o}\left(x, x_{0}\right) \mathrm{d}^{3} x
$$

when occurring in a commutator. Therefore, if there are several "internal" (i.e. $\left.x^{\prime} \equiv x\right)$ SBS transformations present with generating currents as in eq. (2), their charges will obey the same Lie algebra as the corresponding passive transformations. We conclude that in each space-like plane the validity of a charge algebra isomorphic to the Lie algebra of the passive internal invariances is to be expected for the local SBS's.

b) As we saw, the passive SBS transformation changes one irreducible representation of the field algebra into a mathematically inequivalent one. To describe the physics one has to choose one of these solutions. There can be nothing in the original theory which leads to a preference between these descriptions, because this would contradict the assumed passive invariance; the physics must be equally well described by each of the representations. Consequently the original theory can only be a useful one if any quantity which can be uniquely measured*, is represented by the same operator in each of the inequivalent representations of the theory $\dagger$. In other words, it must be possible to define the mentioned observables in such a way that they are invariant under the SBS transformation; only "unobservable" quantities reallt change by the passive transformation.. This is in fact a quite general property of theories with a passive invariance.

Now suppose, for example, that in a SBS theory of the present local type one would have constructed a phenomenological field operator which interpolates well between the out and in fields, corresponding to a bound state of some particles belonging to basic fields. Because the SBS transformation is not unitarily implementable, the asymptotical fields and therefore also the corresponding phenomenological field operators constructed in different inequivalent representations will in general not be linearly related. Nevertheless their transformations must obey the composition rules of the group, because this is the case for the passive transformations of the complete inequivalent descriptions into each other. If, in a classical approximation, it is assumed that the transformed phenomenological fields are simply functions of the original fields at one space-time point, then a passive SBS transformation group is realized non-linearly by the transformations of the phenomenological

* These are the type of quantities which one, for example, may find in tables; they represent a smaller class than one usually defines in quantum mechanics as observables because, as is shown in the text, only scalars with respect to every (spontaneously broken or good) passive invariance transformation belong to it.

$\dagger$ We are considering the different inequivalent representations to be realized in one fixed Hilbert space. 
fields. Furthermore, as remarked earlier, all the observables have to be invariant under these non-linear transformations. If the observables are constructed from a phenomenological Lagrangian it is therefore natural to demand that such a Lagrangian itself should be invariant under the, in general non-linear, realization of the SBS transformations by the occurring fields.

Comparing these points with the actual situation [23-28] in elementary particle physics, it appears that the present class of SBS's has properties which agreee with the ones of the observed approximate symmetries and therefore we shall come back to it in more detail in sect. 3 .

We now consider the case which is nearly exclusively discussed in the literature, namely the one in which the SBS current is even conserved. This assumption is so strong that it is difficult to prevent the symmetry from becoming an unbroken one, as is expressed by Goldstone's theorem. It can be easily shown [6,20] that eq. (2) is now still valid if one takes the time argument of the field operators different from the one of the charge

$$
\lim _{V \rightarrow \infty}\left[\int_{V} j^{\mathrm{o}}\left(\boldsymbol{x}, x_{\mathrm{o}}\right) \mathrm{d}^{3} x, \phi\left(y, y_{\mathrm{o}}\right)\right]=-\left.i \frac{\partial \phi^{\prime}\left(\boldsymbol{y}, y_{\mathrm{o}}\right)}{\partial \alpha}\right|_{\alpha=0},
$$

where again $\phi$ and $\phi^{\prime}$ are the same algebraic expressions in $\psi$ respectively $\psi^{\prime}$. In other words the passive symmetry represents an automorphism for the whole of the quasi-local field algebra. The symmetry can only be broken because there are global operators which do not transform "correctly", as a result of the limiting procedure in eq. (3). We shall therefore call this case the one of asymptotical symmetry breaking. It is the case of the Goldstone bosons or long range interactions. This property, in addition to the presence of a conserved current, disqualifies, in our opinion, the asymptotical type of SBS for a description of the approximate symmetries in elementary particle physics.

It is perhaps of interest to see the fundamental physical difference between local and asymptotical breaking by comparing the physical effects of the locally applied active SBS operations in examples of the two cases. In the well-known model for the asymptotical breaking, the Heisenberg ferromagnet [29], the rotation invariance is spontaneously broken. If one rotates all dipoles in a finite volume of the magnet through a certain angle and leaves them subsequently free, the "excited state" will decay by spin oscillations, starting from the edge of the volume. By taking the rotated region sufficiently large, one is able to keep the centre of this region static during an arbitrarily long time. This possibility to actively perform at least locally the passive invariance transformation is a manifestation of the fact that the physical (unitary) operator reproduces the passive invariance transformation in an arbitrarily large four-dimensional volume.

Compare this with a typical case of local breaking, as the one of the $\mathrm{U}_{2} \otimes \mathrm{U}_{2}$ group in the model of the QED of massless electrons and muons [13,14]. Here, it is not possible during a finite time to obtain a state which is locally stable in which 
the electron is partially transformed into a muon, essentially because the field equations of these particles contain time derivatives which do not change under the unitary transformation as they do under the passive invariance transformation; this reflects the fact that the physical (unitary) transformation is only equivalent to the passive invariance transformation at one time (see eq. (2)). The same is true for spontaneously broken dilatation invariance; one cannot locally dilate the physical world during a finite time! The examples show how close the asymptotically broken symmetries are to the good ones, in which the actively transformed state can be indefinitely maintained, and how unphysical the usual active interpretation of a locally broken symmetry is. This may partially explain the much larger attention the asymptotically broken symmetries have received in the literature, in spite of the fact that they are evidently not relevant for the description of nearly all approximate symmetries in elementary particle physics.

\section{LOCAL SYMMETRY BREAKING}

We have seen that the class of SBS theories in which the symmetry is locally broken has several properties which agree with the present state of our knowledge in elementary particle physics:

(a) The SBS currents are not conserved (which also renders Goldstone's theorem unapplicable).

(b) A charge algebra isomorphic to the Lie algebra of the passive internal transformations emerges.

(c) The passive invariance transformation for phenomenological field operators is in general non-linear. A phenomenological Lagrangian should be invariant under these transformations.

(d) It becomes understandable why the physical states are "stiff" against attempts to apply the passive invariance operation actively, which is one of the prominent features of the observed approximate symmetries in elementary particle physics.

The question then arises whether the assumptions which distinguish the local breakings from the other ones are compatible with each other. This would for example not be the case if Noether's theorem would apply.

We will now construct explicitly a model which fulfills our conditions. Consider a four-component fermion field $\psi_{\alpha}(x)$ obeying the equation

$$
\lim _{V \rightarrow 0} \int_{V} F_{\alpha \beta}\left(\psi ; x-x^{\prime}\right) \psi_{\beta}\left(x^{\prime}\right) \mathrm{d}^{4} x^{\prime}=0,
$$

and the canonical equal time commutation rules

$$
\begin{aligned}
& \left\{\psi_{\alpha}(x), \psi_{\beta}^{+}(y)\right\}=\delta_{\alpha \beta} \delta^{3}(x-y) \\
& \left\{\psi_{\alpha}(x), \psi_{\beta}(y)\right\}=\left\{\psi_{\alpha}^{+}(x), \psi_{\beta}^{+}(y)\right\}=0 .
\end{aligned}
$$


Here $F_{\alpha \beta}\left(\psi ; x-x^{\prime}\right)$ is a distribution which is defined as the functional of the field $\psi$ given by the inverse of its propagator, which is assumed to have a positive-definite Källén-Lehmann density:

$$
-i \int F_{\alpha \beta}\left(\psi ; x-x^{\prime \prime}\right)\left\langle 0\left|\mathrm{~T}\left\{\psi_{\beta}\left(x^{\prime \prime}\right) \bar{\psi}_{\gamma}\left(x^{\prime}\right)\right\}\right| 0\right\rangle \mathrm{d}^{4} x^{\prime \prime}=\delta_{\alpha \gamma} \delta^{4}\left(x-x^{\prime}\right)
$$

The limit $V \rightarrow 0$ means that the volume over which the integration is performed tends to zero, always including the point $x$. By this limiting procedure the field equation is manifestly local.

It is easy to see that the theory defined by the eqs. (4a), (4b) and (5), is passively invariant under the transformation

$$
\psi^{\prime}(x)=\mathrm{e}^{i \theta \gamma 5} \psi(x)
$$

One finds

$$
F\left(\psi^{\prime} ; x-x^{\prime \prime}\right)=\mathrm{e}^{-i \theta \gamma 5} F\left(\psi ; x-x^{\prime \prime}\right) \mathrm{e}^{-i \theta \gamma 5},
$$

and therefore if $F(\psi) \psi=0$ also $F\left(\psi^{\prime}\right) \psi^{\prime}=0$. As $\psi^{\prime}$ obeys also the commutation rules (4b) it is a new solution of the theory and transformation (6) is by definition a passive symmetry.

It is also clear that the chiral transformation (6) is generated in the sense of eq. (2) by the local current

$$
j_{5}^{\mu}(x)=\bar{\psi}(x) \gamma^{\mu} \gamma_{5} \psi(x)
$$

which will turn out to be in general not conserved. Therefore our model fulfills all the conditions of a local SBS theory.

Furthermore, the usual free field is a particular SBS solution of the theory. One finds by Fourier transforming twice eq. (5) for this case

$$
F\left(\psi_{0} ; x-x^{\prime}\right)=\left(i \partial_{x}-m\right) \delta^{4}\left(x-x^{\prime}\right)
$$

and that consequently the field equation ( $4 a$ ) is fulfilled, because it is now just the Dirac equation defining the free field.

By applying the passive invariance transformation (6), one obtains a continuous set of inequivalent solutions. In each representation (characterized by the unobservable parameter $\theta$ ) the field equation ( $4 a$ ) is equivalent to a generalized Dirac equation

$$
\left(i \not-m \cos 2 \theta+i m \gamma_{5} \sin 2 \theta\right) \psi(\theta ; x)=0 .
$$

Eq. (9) plays in our model the role which a phenomenological equation would play 
in a realistic SBS theory. Such equations with a fixed value of the unobservable "phase" are only valid in one of the possible inequivalent representations and are, of course, in general non-invariant [3]. As remarked earlier all actually observable quantities are independent of the phase.

The "normal" solution (i.e. the one for which the passive invariance is not broken) is given by the neutrino field. To make the present somewhat trivial theory physically unique one should explicitly state whether the normal or the SBS solution is realized. For the physical uniqueness of our model see also the appendix.

We now list some properties of the model which partially have a more general relevance.

The passive transformation of an operator not constructed from the $\psi$ 's in one space-like plane, as for example $\partial \psi / \partial x_{0}$, is not generated by the $j_{5}^{\mu}$ current. One may verify that this fact is directly related to the current non-conservation.

It can be readily shown that the dilatational invariance is also a local SBS for the solutions just discussed. By applying the passive dilatation transformation on a solution one generates a new manifold of mathematically inequivalent representations characterized by the mass value. Physically these representations are equivalent because the absolute mass scale is not measurable [9] .

By giving the field the additional degrees of freedom of quark fields, the discussion of the $\gamma_{5}$-invariance can be generalized to the one of the U(3) $\otimes U(3)$ chiral group*; one finds of course the validity of the U(3) $\otimes U(3)$ charge algebra although the currents may in part not be conserved.

Where does the proof of Noether's theorem fail in the present case? Going through this proof one sees that an essential point is that the passive invariance transformation has the same effect on the Lagrangian as does the local variation of the field. The Lagrangian density for our case would be

$$
\mathcal{L}(x)=\lim _{V(\epsilon) \rightarrow 0} \frac{1}{2} \int\left\{\bar{\psi}\left(x+\frac{1}{2} \epsilon\right) F(\epsilon) \psi\left(x-\frac{1}{2} \epsilon\right)+\text { C.C. }- \text { V.E.V. }\right\} \mathrm{d}^{4} \epsilon
$$

If the field is locally varied $F$ does not change**. However under a passive transformation, because this transformation extends over the whole of space-time, $F$ does change. This difference between the effects of the local field variation and the passive transformation results in the breakdown of the proof of Noether's theorem.

* In fact even to a $\mathrm{U}(12)$ group; as the dilatations, $\mathrm{U}(12)$ can be an exact passive invariance transformation in a theory with finite masses!

** This is necessary for the Lagrangian (10) to produce our field equation and follows from the c-number character of the variations. If one has doubts about this point, one may also think the propagator in eq. (5) to be defined (similarly as in ref. [3]) as being the quantity

$$
\overline{\mathrm{T} \psi_{\beta}(z+\epsilon) \overline{\psi_{\gamma}(z)}},
$$

the bar meaning an average over all values of $z$ in an infinite space-time volume. 
The just discussed model proves the consistency of the definition of a local SBS but it may seem to be somewhat artificial. We believe that this is not the case and we shall now briefly indicate how the model may reflect the connection between a passive invariance, its spontaneous breaking and the renormalizations necessary in a realistic theory. In fact this was the way we arrived at the model.

Replacing in the Lagrangian (10) $\bar{\psi}\left(x+\frac{1}{2} \epsilon\right) \psi\left(x-\frac{1}{2} \epsilon\right)$ by its gauge invariant version

$$
\bar{\psi}\left(x+\frac{1}{2} \epsilon\right) \phi(x, \epsilon) \psi\left(x-\frac{1}{2} \epsilon\right)
$$

with $\phi$ defined as in ref. [30] by

$$
\phi(x, \epsilon)=: \exp \left\{-i e \int_{-\frac{1}{2} \epsilon}^{\frac{1}{2} \epsilon} \hat{\epsilon} \cdot A\left(x+\epsilon^{\prime} \hat{\epsilon}\right) \mathrm{d} \epsilon^{\prime}\right\}:
$$

the resulting field equations are at least very near to the ones of renormalized QED as may be seen by comparison with the formulation of ref. [30]. Up to a $\gamma_{5}$-trans- formation, the inverse propagation is of the type

$$
F_{\mathrm{e}}(\epsilon)=\left\{Z_{\mathrm{e}}\left(\epsilon^{2}\right) i \gamma \cdot \frac{\partial}{\partial \epsilon}-m_{\mathrm{e}}\left(\epsilon^{2}\right)\right\} \delta_{\mathrm{e}}^{4}(\epsilon)
$$

with

$$
\lim _{\mathrm{e} \rightarrow 0}\left\{Z_{\mathrm{e}}\left(\epsilon^{2}\right) ; m_{\mathrm{e}}\left(\epsilon^{2}\right) ; \delta_{\mathrm{e}}^{4}(\epsilon)\right\}=\left\{1 ; m ; \delta^{4}(\epsilon)\right\}
$$

The differentiation now also introduces the interaction whereas the function $Z\left(\epsilon^{2}\right)$ takes care of the infinite renormalizations needed because the product of interaction field operators is more singular than the one of the free operators; by its definition (5) it is clear that $F$ tends to compensate this singularity. As in refs. [30] and [31], $Z(0)$ has the meaning of the usual renormalization constants $Z_{1}$ and $Z_{2}$ and may now vanish without creating any inconsistency. The term $m\left(\epsilon^{2}\right)$ was not included in the treatment of ref. [30] and is a self consistent mass subtraction term. We remark at the side that an approximate calculation shows that $m(0)=0$ and that $m\left(\epsilon^{2}\right)$ plays just the role of $d(\epsilon)$ in eq. (6) of ref. [19].

Here (or in the formulation of refs. [30,31]) one may now think the non-local theory for finite $V$ (resp. $\epsilon$ ) to be solved first and assume that the solution of the local theory is obtained by going in the non-local solutions with $V \rightarrow 0$ (resp. $\epsilon \rightarrow 0$ ). For finite $V$ (resp. $\epsilon$ ) there is a passive $\gamma_{5}$-invariance; the proof is the same as for our earlier free field model, except that now the non-vanishing commutation rules are not demanded to be canonical, but are selfconsistently determined by the propagators. It follows that also the limits of the solutions for $V \rightarrow 0$ (resp. $\epsilon \rightarrow 0$ ) are 
connected by the $\gamma_{5}$-transformation, which shows the passive $\gamma_{5}$-invariance of the local theory. For a generalization to the unitary transformations in the case of the QED of more lepton fields, these passive invariances demand that the cutoff volume $V$ (resp. $\epsilon$ ) be the same for all lepton fields. We hope to come back to this type of theory in more detail in a future publication.

In general the renormalizations in quantum field theories have the same bootstrap-like property as $F\left(\psi ; x-x^{\prime}\right)$ does through eqs. (4a) and (5), namely that they define the theory, but are themselves again implicitly determined by the solution of the theory. This is the case whether the renormalizations are multiplicative or subtractive, infinite or finite. If there are inequivalent solutions, in most cases the renormalizations will depend on the solution chosen. In a correct formulation the renormalization should therefore transform automatically along with the fields, if these are passively transformed. This causes the fact that the passive invariance transformation of the local field equations (or Lagrangian) is not achieved by only applying a unitary transformation to them; just this situation is reproduced in our model, resulting in a SBS with a non-conserved symmetry current.

One may note that if the dependence of the renormalization on the fields is explicitly included in the Lagrangian or the field equations any finite renormalization

$$
\psi^{\prime}=Z^{\frac{1}{2}} \psi
$$

is a passive invariance transformation as, for example, can be seen in eqs. (4a) or (10). By combining transformations (11) and (1), a certain freedom in the choice of relation (1) is created*. For relation (2) to be possible the equal time commutation rules of the transformed fields (or more generally, the leading small distance singularity of its propagators) should be equal to the ones of the original fields, and this fixes the relative normalizations of the sets $\psi$ and $\psi^{\prime}$ and therefore also the passive transformation (1), which turns out to be often but not always identical to the transformation of the unrenormalized fields.

An interesting exception leads to the so called anomalous dimensions [32]. As was remarked nearly ten years ago (refs. $[9,10]$ ) the dilatation may be considered as the prototype of a SBS. From experience one knows that quite generally in the description of a physical system:

(1) A change of the unit of length leads to a new description of the same physics.

(2) Such a change cannot be achieved by a unitary operation, because one can in general not actually dilate the states of a realistic system.

For a theory of which the solutions reproduce these basic features, the dilatation is by our definition a SBS.

Can the dilatation be a local SBS? Suppose the dilatation transformation and the renormalization (11) are both passive invariances which are spontaneously broken.

\footnotetext{
* In our model this choice does not exist because of the normalized commutation rules (4b), but in a theory of interacting fields such a fixed normalization will in general not occur.
} 
Which one of all the possible combinations of these transformations can be generated by a local current? This would be the one for which the asymptotic behaviour (for small $x$ or large $p$ ) of the propagator is invariant. For a fermion propagator which goes at high momenta as the free one multiplied, as in ref. [31], by a power of the type $\left(m^{-2} p^{2}\right)^{\beta}$ one easily finds that to keep this behaviour invariant a dilatation

$$
\psi^{\prime}(x)=\lambda^{\frac{3}{2}} \psi(\lambda x)
$$

has to be accompanied by a renormalization

$$
\psi^{\prime \prime}(x)=\lambda^{\beta} \psi^{\prime}(x)
$$

The total transformation is

$$
\psi^{\prime \prime}(x)=\lambda^{\frac{3}{2}+\beta} \psi(\lambda x)
$$

with $\beta$ being the anomalous dimension.

One may even show more, namely that, if a combination of the normal dilatation and a finite renormalization is a local SBS, then the leading singularity of a product of basic field operators has a power behaviour. Furthermore it is clear that any quantity which is renormalization invariant (e.g. a conserved current) has its normal dimension.

We remark that even though the propagators of $\psi$ and $\psi^{\prime}$ have the same behaviour and normalization for infinite momenta, in the SBS case they will at low momenta have different normalizations and singularities. The asymptotic symmetry of the propagators is typical for a local SBS; at sufficiently small distances (high momenta) the broken passive invariance is recovered. These points may be verified for the dilatations and $\gamma_{5}$-transformations in our model.

The mechanism which causes the self-consistent local symmetry breaking is evidently one in which a global property of the solution (as the renormalization is) has a local effect. One might try to reproduce such a situation in a classical model. For example, introducing in the Hamiltonian of the Heisenberg ferromagnet [29] the interaction term $\langle 0|\boldsymbol{\sigma}(\boldsymbol{x})| 0\rangle \cdot \boldsymbol{\sigma}(\boldsymbol{x})$, one will have maintained the passive rotational invariance but self-consistently broken the spin current conservation, because the expectation value of the spin is fixed by the dipole direction at infinite. However this type of interaction seems in a classical model physically only to occur as a result of a long range interaction; in the example of the ferromagnet this could be the homogeneous overall magnetic field stemming from the far away dipoles. As the model given earlier directly shows, in a quantum field theory no long range interaction is necessary for a global property to affect the local field equations. 


\section{REMARKS}

Finally we would like to make the following loosely connected remarks:

(1) The reason why SBS's are at all observed is the fact that in certain limiting situations the passive symmetries can emerge as good physical ones. For example in the model of massless QED, the spontaneously broken dilatational invariance becomes at high momenta relatively good. The fermion propagator in the Fermi gauge $[30,31]$ deviates from the dilatationally invariant one only by an extra factor $\left(m^{-2} p^{2}\right)^{\alpha / 4 \pi}$. Similarly the physical electron-muon symmetry gets restored at sufficiently high momenta. In spite of the absence of a dynamical theory, it appears that both with respect to asymptotic dilatational [32] and chiral [33] invariance the general situation in hadron physics is somewhat comparable to the just mentioned one of the leptons. In contradistinction to the point of view taken in ref. [32] it seems to us that, for the understanding of the generally observed approximate dilatational invariance in elementary particle physics, its SBS character is essential; in a halfway realistic theory exact passive dilatational invariance cannot be represented by a unitary operator which leaves the vacuum invariant.

(2) We would like to sketch the relation of our approach to the one which introduces a skeleton theory [32]. To begin with, it is easy to see that certain approximate invariances as dilatation invariance and $\mathrm{SU}(6)$, cannot be exact physical invariances (i.e. represented by unitary transformations) in theories which resemble true physics (which, for example, have a discrete non-trivial mass spectrum). To give these invariances an exact meaning, an unphysical element must come in. One could think of postulating the existence of a skeleton theory (which necessarily is quite unphysical) for which the mentioned invariances are exact "physical" ones, i.e. are represented by unitary transformations. The real physical theory is then supposed to be generated from the skeleton theory by the introduction of suitably chosen symmetry breaking perturbations.

In our approach we give up considering the approximate invariances as active physical ones in any sense; they are exact mathematical passive invariances, related to the existence of a manifold of inequivalent solutions of the theory. As passive invariances, they are not in contradiction with the physical properties of the theory nor is there any problem with their coexistence as was already shown in our model. We have seen that when the passive transformations are generated by local currents, this guarantees the physical relevance of the passive invariances at very small distances or high momenta. Because of the assumed passive invariance, no symmetry breaking parameter needs to be present in the basic theory. Therefore the type and amount of the deviations of the physical symmetries from the exact ones should in this case in principle not be introduced ad hoc (as in skeleton theories) but be determined selfconsistently, as one already can do in QED for the breaking of the $\gamma_{5^{-}}$ symmetry and dilatation invariance and partially for the breakings of the $\mathrm{SU}(2) \otimes$ $\mathrm{SU}(2)$ symmetries in the QED of electrons and muons $[13,14]$. From our point of view, even in the unlikely case that a kind of skeleton theory could be constructed, 
the realistic situation would be related to the skeleton solution in the same way as a superconductive state is related to the normal one [2], i.e. non-analytically and therefore not perturbatively.

(3) The present day state of the art in quantitatively treating quantum field theories does not allow one to make directly use of the SBS idea in most cases of hadron physics; up to now one cannot do much better than to add a symmetry breaking term to the Lagrangian and calculate in lowest order. What we have shown in the present paper is that quantum field theory is not a priori.unsuitable to provide a quantitative understanding of the symmetry breakings. On the contrary, the renormalization is a built-in mechanism which can produce selfconsistent symmetry breakings of the observed type, i.e. with non-conserved currents and without Goldstone bosons.

(4) In hadron physics the approach which considers the pseudoscalar mesons as a kind of approximate Goldstone bosons with finite mass [2] has been surprisingly successful. Similar bosons do not occur in the SBS approach to QED and the question seems therefore to demand special dynamical considerations. The lowest mass value of the states which are created by applying a generator of the passive invariance transformation on the vacuum, may be considered as a measure for the earlier discussed stiffness of the system against the local SBS transformation. In this sense the low pion mass shows that one may not be too far from the limit in which the passive chiral symmetry operation is locally implementable.

(5) General conformal invariance, in particular the inversion, gives difficulties as a finite passive invariance transformation because it contains a singular space-time point. Therefore only infinitesimal conformal broken symmetry transformations $[34,8]$ are considered in practice. For the same reason general conformal invariance cannot be a SBS in the sense of the present paper. As in any realistic system also the conformal current is not conserved, we do not know of any definition by which general conformal invariance could be a spontaneously broken symmetry.

That there is at least a basic difference between the dilatation and the more general conformal transformations in this respect can be observed nearly immediately. As we saw, the presence of a SBS demands the possibility of different descriptions in which observable quantities have the same values, and which are related to each other by the passive invariance transformation. As remarked earlier, with respect to dilatational invariance these descriptions are obviously always present; they correspond just to different choices of the unit of length. This is probably the most direct argument for the general relevance of spontaneously broken dilatational invariance to elementary particle physics $[9,10]$. In contradistinction to this case, it seems that there does not exist any general change of description which corresponds to an inversion or a special conformal transformation.

(6) The discussed types of symmetries range from the only mathematical one to the bonafide physical symmetry, i.e., the one which can be completely actively interpreted. Whether one speaks of spontaneous breaking of a physical symmetry or of self-generation of such a symmetry, depends on the direction in which one goes through the classification. 
(7) One might ask what can be the meaning of a mathematical symmetry if it is not physically realized? The question seems to be in timately related with the more general one: on which basis should one hope for, or even expect, a basic theory to be mathematically simple, as physical phenomena are evidently complicated? An interesting discussion of this fundamental problem is given in ref. [35]. Anyhow, if the present interpretation of the observed approximate symmetries is true, then the corresponding passive invariances would play a similar role as Lorentz invariance and other good invariances in limiting the possibilities for the construction of a basic theory and could allow for a derivation of the physical symmetry breakings.

We thank J.A.Swieca for helpful discussions. One of us (T.M.) is grateful to the members of the Theoretical Group at DESY for their kind hospitality and in particular to R.Haag and K.Symanzik for stimulating discussions during a two-month stay. He is indebted to R.Haag and H.Joos for making this visit possible through the Brazilian-German Scientific and Technological Agreement.

\section{APPENDIX}

Uniqueness of the free field solution of our model

From our field equation (4a) follows

$$
\int_{\substack{V \rightarrow 0 \\\left(x, x^{\prime} \text { in } V\right)}} F\left(x-x^{\prime}\right) S\left(x^{\prime}\right) \mathrm{d}^{4} x^{\prime}=0,
$$

where

$$
S\left(x^{\prime}\right)=\left\langle 0\left|\psi\left(x^{\prime}\right) \bar{\psi}(0)\right| 0\right\rangle
$$

Furthermore by definition (5), assuming $\gamma_{\mathrm{o}} F_{\beta \alpha}^{*}(-x) \gamma_{\mathrm{o}}=F_{\alpha \beta}(x)$

$$
\begin{gathered}
\int F\left(x-x^{\prime}\right) S_{\mathrm{F}}\left(x^{\prime}\right) \mathrm{d}^{4} x^{\prime}=\delta^{4}(x), \\
\int F\left(x-x^{\prime}\right) S_{1}\left(x^{\prime}\right) \mathrm{d}^{4} x^{\prime}=0,
\end{gathered}
$$

(the integrals are over the whole of $x$-space).

For eqs. (A.1) and (A.2) to be fulfilled, the distribution $F$ must have the property that its operation is not affected by the $V$-cutoff. This is just the self consistency condition which is described in the text as applied to this case. This very strong demand is fulfilled if $F(y)$ is a distribution localized at $y=0$. In this case, which seems to be the only possibility, one has

$$
\tilde{F}(p)=P\left(p^{2}\right) p p+Q\left(p^{2}\right) \gamma_{5} p+R\left(p^{2}\right)+U\left(p^{2}\right) \gamma_{5},
$$


where $P, Q, R$ and $U$ are polynomials of finite degree. One has then

$$
\tilde{S}(p)=\tilde{F}^{-1}(p)
$$

Except when $P, Q, R$ and $U$ are constants, this propagator would be less singular than the free one, in contradiction to the positive definiteness of the Källén-

Lehmann density and the assumed commutation rules, which even demand $P=1$, $Q=0$. This gives our solutions.

\section{REFERENCES}

[1] W.Heisenberg, Rev. Mod. Phys. 29 (1957) 269.

[2] Y.Nambu and G.Jona-Lasinio, Phys. Rev. 122 (1961) 345.

[3] R.Haag, Nuovo Cimento 25 (1962) 287.

[4] J.Goldstone, A Salam and S.Weinberg, Phys. Rev. 127 (1962) 965.

[5] A.Katz and Y.Frishman, Nuovo Cimento 42A (1966) 1009.

[6] D.Kastler, D.W.Robinson and J.A.Swieca, Com. Math. Phys. 2 (1966) 108.

[7] H.Ezawa and J.A.Swieca, Com. Math. Phys. 5 (1967) 330.

[8] C.J.Isham, A.Salam and J.Strathdee, Broken chiral and conformal symmetry in an effective Lagrangian formalism, I.C.T.P. preprint (1970).

[9] Th.A.J.Maris, Nuovo Cimento 30 (1963) 378.

[10] R.Haag and Th.A.J.Maris, Phys. Rev. 132 (1963) 2325.

[11] M.Baker and S.L.Glashow, Phys. Rev. 128 (1962) 2462.

[12] K.Johnson, M.Baker and R.S.Willey, Phys. Rev. 136 (1964) B1111.

[13] Th.A.J.Maris, V.E.Herscovitz and G.Jacob, Nuovo Cimento 34 (1964) 946.

[14] R.Arnowitt and S.Deser, Phys. Rev. 138 (1965) B712.

[15] Th.A.J.Maris, G.Jacob and B.Liberman, Nuovo Cimento 52A (1967) 116.

[16] K.Johnson, in Proc. of the 9th Latin American school of physics, edited by I.Saavedra (Benjamin, 1968).

[17] This expression has been used by A.Pais for a similar situation.

[18] K.Johnson, Phys. Letters 5 (1963) 253.

[19] Th.A.J.Maris, Nuovo Cimento Letters 2 (1969) 821.

[20] R.F.Streater, Proc. Roy. Soc. A287 (1965) 510.

[21] G.S.Guralnik, C.R.Hagen and T.W.B.Kibble, in Advances in particle physics, edited by Cool and Marshak (Interscience, 1968).

[22] J.Lowenstein and J.A.Swieca, Quantum electrodynamics in two dimensions, preprint, Inst. Física, Univ. of São Paulo (1970).

[23] M.Gell-Mann, Physics 1 (1964) 63.

[24] S.L.Adler and R.F.Dashen, Current algebras and applications to particle physics (Benja$\min , 1968)$.

[25] G.Kramer, H.Rolinik and B.Stech, Z. Phys. 154 (1959) 564.

[26] S.Weinberg, Phys. Rev. Letters 18 (1967) 188.

[27] J.Schwinger, Phys. Letters 24B (1967) 473.

[28] F.Gürsey, in Particles, currents, symmetries, edited by P.Urban (Springer, 1968).

[29] W.Heisenberg, Z. Phys. 49 (1928) 619.

[30] Th.A.J.Maris, D.Dillenburg and G.Jacob, Nucl. Phys. B18 (1970) 366.

[31] D.Dillenburg and Th.A.J.Maris, Nucl. Phys. B 18 (1970) 390. 
[32] K.Wilson, Operator product expansions and anomalous dimensions in the Thirring model, and A model of coupling constant renormalization, SLAC preprints (1970).

[33] T.Das, V.S.Mathur and S.Okubo, Phys. Rev. Letters 18 (1967) 761.

[34] H,A.Kastrup, Ann. der Phys. 9 (1962) 388.

[35] E.P.Wigner, Symmetries and reflections (Indiana University Press, 1967). 